\title{
3 Research Square

\section{Preoperative predictive factor analysis of ovarian malignant involvement in premenopausal patients with clinical stage I endometrioid endometrial carcinoma}

\section{Qin Chen}

Zhejiang University School of Medicine

\section{Yan Feng}

Zhejiang University School of Medicine

\section{Wenwen Wang}

Zhejiang University School of Medicine

\section{Weiguo Lv}

Zhejiang University School of Medicine

\section{Baohua Li ( $\square$ lbh19787@zju.edu.cn )}

Zhejiang University School of Medicine Women's Hospital https://orcid.org/0000-0002-2205-3248

\section{Research article}

Keywords: clinical stage I endometrioid endometrial carcinoma, preoperative predictive factors, ovarian malignant involvement, premenopausal patients

Posted Date: January 7th, 2020

DOI: https://doi.org/10.21203/rs.2.17501/v2

License: (c) (i) This work is licensed under a Creative Commons Attribution 4.0 International License. Read Full License

Version of Record: A version of this preprint was published at Scientific Reports on January 13th, 2021. See the published version at https://doi.org/10.1038/s41598-020-78953-4. 


\section{Abstract}

Background Earlier literature suggests that ovarian preservation in young premenopausal clinical stage I endometrioid endometrial carcinoma patients does not negatively impact prognosis and is a more suitable choice for management of the disease. The main purpose of this study was to clarify the incidence of ovarian malignant involvement in premenopausal clinical stage I endometrioid endometrial carcinoma and further identify potential preoperative predictive factors of ovarian malignant involvement. Methods Premenopausal patients ( $\leq 50$ years) with clinical stage I endometrioid endometrial carcinoma subjected to total hysterectomy and bilateral salpingo-oophorectomy with or without pelvic and/or para-aortic lymph node dissection at Women's Hospital, Zhejiang University School of Medicine between 2002 and 2016 were enrolled for study. Patients were excluded in cases of gross extra pelvic disease on examination or imaging and family history of colon or gastrointestinal carcinoma. The included patient population was examined for incidence of ovarian malignant involvement and potential preoperative clinical predictive factors.Results A total of 511 premenopausal (age $\leq 50$ years) patients diagnosed with clinical stage I endometrioid endometrial carcinoma were enrolled for the study. Ovarian malignant involvements were detected in 23 of the patients (4.5\%). Kaplan-Meier analysis showed poorer prognoses of patients with ovarian malignant involvement than those without ovarian involvement. Univariate and multivariate logistic analysis validated preoperative imaging of myometrial invasion depth, the gross appearance of the ovaries, and preoperative serum carbohydrate antigen 125 (CA125) level as independent risk predictors of postoperative ovarian malignant involvement. Receiver operating characteristic (ROC) curves was individually generated for preoperative myometrial invasion depth, the gross appearance of the ovaries, and serum CA125 level as well as a combination of the three factors. The area under curve (AUC) was 0.858 (95\% confidence interval [CI], 0.757-0.960) for the combined three factors.Conclusions The incidence of ovarian malignant involvement in premenopausal patients with clinical stage I endometrioid endometrial carcinoma was relatively minimal. Preoperative imaging of myometrial invasion depth, the gross appearance of the ovaries, and serum CA125 level were independent risk predictors of ovarian malignant involvement. These findings may facilitate preoperative counseling of patients and informed clinical decision-making on ovarian preservation in these patients.

\section{Background}

Endometrial carcinoma is one of the most common gynecological malignancies prevalent in postmenopausal females in the sixth and seventh decades of life (median age of onset, 60 years) [1]. The standard surgical approach for endometrial carcinoma includes total hysterectomy and BSO with or without pelvic and/or para-aortic lymph node dissection, according to the existence of high-risk factors [13]. At present, the incidence of young premenopausal women with endometrial carcinoma is gradually increasing, with $5-30 \%$ women aged $\leq 50$ years at the time of diagnosis [4]. In the majority of young premenopausal patients, endometrioid endometrial carcinoma is the most common endometrial carcinoma subtype and is usually confined to clinical stage I, namely, located within the corpus uteri 
without malignant involvement extending beyond the uterus and metastasis to the pelvic and/or paraaortic lymph nodes $[4,5]$.

Oophorectomy is commonly performed in young premenopausal women with endometrioid endometrial carcinoma in conjunction with hysterectomy, as an estrogen-responsive tumor. In terms of therapeutic benefits, oophorectomy not only decreases estrogen production but also eliminates the occult co-existing involved ovarian metastasis. However, a surgical pathological study for clinical stage I endometrial carcinoma conducted by the Gynecologic Oncology Group reported only a 5\% incidence of adnexal involvement, especially in young premenopausal women [6]. The incidence of isolated microscopic ovarian involvement was also uncommon $(\sim 1 \%)$ [7]. In addition, estrogen deprivation resulting from oophorectomy in young premenopausal women may cause a rapid decline in circulating ovarian estrogen and androgens, leading to increased short- and long-term adverse outcomes, including hot flushes, vaginal atrophy, sleep disorders, cardiovascular disease, osteoporosis, dementia, cognitive impairment, depression and anxiety as well as permanent loss of fertility [3]. Use of this traditional surgical approach is controversial due to occasional ovarian involvement and adverse sequela of estrogen deprivation in young premenopausal patients with clinical stage I endometrioid endometrial carcinoma. However, the safety and feasibility of ovarian preservation in the patient group is of widespread concern. To our knowledge, limited studies have focused on preoperative predictive factors of ovarian malignant involvement in these patients, which may be utilized to determine the optimal surgical approaches for providing maximal therapeutic benefits and further distinguish patients requiring BSO.

Here, we performed a retrospective study on premenopausal patients with clinical stage I endometrioid endometrial carcinoma admitted in Women's Hospital, Zhejiang University School of Medicine, between January 2002 and December 2016. The incidence of ovarian malignant involvement in these patients and possible preoperative predictive factors were further determined, with a view to optimizing therapeutic management.

\section{Methods}

This retrospective study was performed on premenopausal patients (age $\leq 50$ years) with clinical stage I endometrioid endometrial carcinoma subjected to total hysterectomy and BSO with or without pelvic and/or para-aortic lymph node dissection from Women's Hospital, Zhejiang University School of Medicine, between January 2002 and December 2016. Patients were identified using hospital tumor registries and an internal database of gynecological carcinoma cases. During this period, 1,978 patients, who were diagnosed with endometrial carcinoma, were admitted in our hospital. CT/MRI imaging was performed to examine myometrial invasion depth and the presence or absence of enlarged or suspicious paraortic and pelvic nodal disease as well as adnexal involvement. Basis on the preoperative imaging results, patients with suspicious gross extra uterus metastasis were excluded. Patients were also excluded with family history of colon or gastrointestinal carcinoma. Finally, the included patients with clinical stage I endometrioid endometrial carcinoma were 1,228 cases. Among them, the numbers of premenopausal patients (age $\leq 50$ years) were 522. Basically, the enrolled patients did not receive 
preoperative adjuvant treatment\on account of premenopausal clinical stage I endometrioid endometrial carcinoma management option.

All pathological sections were confirmed by two gynecological pathologists (QC and WWW). Ovarian malignant involvement was further determined via postoperative pathologic examination. Among the 522 patients examined, 34 displayed ovarian malignancy involvement, specifically, ovarian endometrioid carcinoma (23 cases), synchronous primary uterine and ovarian tumors (4 cases), borderline tumor (3 cases), serous papillary carcinoma (2 cases), clear cell carcinoma (1 case) and mucinous adenocarcinoma (1 case). Patients with endometrioid histology of ovary and uterine were included, while other pathological subtypes and independent synchronous primary uterine and ovarian tumors were excluded, according to the pathological criteria of Ulbright and Roth in 1985 [8] used to distinguish ovarian malignant involvement from independent synchronous endometrial and ovarian carcinoma. Furthermore, an extensive pathological characteristic formulated by Scully et al. [9] was applied to differentiate between uterine endometrial carcinoma with ovarian malignant involvement and independent synchronous primary uterus and ovarian carcinoma. Clinicopathological characteristics favoring primary uterine endometrial tumors with ovarian malignant involvement included histological similarity of tumors, uterine larger size of tumor, presence of uterine atypical endometrial hyperplasia and deep myometrial invasion $(\geq 1 / 2)$ with direct extension into adnexa and/or lymphovascular invasion. And other evidence of spread from uterine endometrial tumor included bilateral, multinodular, surface involvement of the ovary and absence of endometriosis [9]. Finally, 511 premenopausal patients $(\leq 50$ years) with clinical stage I endometrioid endometrial carcinoma were selected for statistical analysis, according to the above admittance standards. Patient demographics and clinicopathological data were obtained through hospital electronic medical records systems and paper charts $₫$ Table $1 \bigotimes$.

All 511 patients were divided into low risk $(n=193)$ and sub-high risk groups $(n=318)$ according to the degree of endometrioid endometrial cancer differentiation and/or deep ( $\geq 1 / 2)$ myometrial invasion [10]. Pre-operative clinicopathological factors were used to evaluate the likelihood of postoperative ovarian malignancy involvement.

All 511 patients enrolled in the study were followed up postoperatively by interview at the clinic or telephone call. Disease-free survival (DFS) and overall survival (OS) rates were calculated from the day of the surgery until recurrence or death. The deadline of follow-up was December 2018. In total, 89 patients $(89 / 511,17.42 \%)$ were lost to follow-up. The mean follow-up time was 94.56 months (range, $12-202$ months). We recorded 26 recurrences $(26 / 422,6.16 \%)$ and 22 deaths $(22 / 422,5.21 \%)$ during this period.

Statistical analysis was performed using SPSS version 23.0. Kaplan-Meier analysis was used to evaluate disease-free and overall survival rate differences between patients with and without ovarian malignant involvement. We applied univariate and multivariate regression models to analyze preoperative predictive factors for ovarian malignant involvement in clinical stage I endometrioid endometrial carcinoma and plotted receiver operating characteristic (ROC) curves. Data were considered significant at $p$-values $<0.05$. 
The present study was approved by the Institutional Review Board of Women's Hospital. Informed consent was obtained from all individual participants.

\section{Results}

A total of 511 premenopausal ( $\leq 50$ years) patients with clinical stage I endometrioid endometrial carcinoma were identified. Among these, 28 patients were poorly differentiated according to preoperative pathological data by endometrial biopsy, and 31 cases had deep myometrial invasion $(\geq 1 / 2)$ by preoperative imaging examination (Table 2). Furthermore, 193 patients from low risk groups were subjected to total hysterectomy and BSO. A further 318 patients identified with at least one risk factor of sub-high risk groups underwent total hysterectomy and BSO with pelvic and/or para-aortic lymph node dissection.

According to postoperative clinicopathological data (Table 1), lymph vascular space invasion involvement (LVSI) was identified in 15 (2.94\%) of 511 cases; lymph node metastasis (LNM) was confirmed in $16(5.03 \%)$ of 318 cases; 41 cases were poorly differentiated; 34 cases had deep myometrial invasion $(\geq 1 / 2)$. For preoperative myometrial invasion depth by imaging examination, true positive rate (TPR), true negative rate (TNR), positive predictive value (PPV), and negative predictive value (NPV) were 52.94\% (18/34), 97.27\% (464/477), 58.06\% (18/31), and 96.67\% (464/480), respectively. For preoperative pathological tumor grade by endometrial biopsy, TPR, TNR, PPV and NPV were 43.9\% (18/41), 97.87\% (460/470), 64.29\% (18/28), and 95.24\% (460/483), respectively. More importantly, the overall ratio of ovarian malignant involvement in 511 patients with clinical stage I endometrioid endometrial carcinoma was $4.5 \%(23 / 511)$, and the counterparts of low risk and sub-high risk patient groups were $1.04 \%(n=2)$ and $6.6 \%(n=21)$. The sub-high risk group was associated with a higher rate of ovarian malignancy involvement $(P=0.003)$ than the low-risk group.

In Kaplan-Meier analysis, patients without ovarian malignant involvement displayed longer disease-free survival $\left(P=8.0 \mathrm{E}^{-6}\right.$, Figure $\left.1 \mathrm{~A}\right)$ and higher five-year survival rates $\left(P=2.61 \mathrm{E}^{-7}\right.$, Figure $\left.1 \mathrm{~B}\right)$ than those with ovarian malignant involvement. Differences were significant between the groups, clearly indicating poorer prognosis of patients with ovarian malignant involvement.

Next, univariate and multivariate regression models were applied to determine preoperative predictive factors for ovarian malignant involvement in clinical stage I endometrioid endometrial carcinoma patients. Univariate logistic analysis disclosed the correlations of preoperative imaging of myometrial invasion depth $\left(P=1.0 \mathrm{E}^{-6}\right)$, the gross appearance of the ovaries $\left(P=1.0 \mathrm{E}^{-5}\right)$, and serum Carbohydrate Antigen 125 (CA125) level $\left(P=3.2 \mathrm{E}^{-5}\right)$ with prediction of ovarian malignant involvement in these patients (Table 2). Multivariate logistic analysis further validated preoperative imaging of myometrial invasion depth $(P=0.005)$, the gross appearance of the ovaries $\left(P=1.27 \mathrm{E}^{-8}\right)$, and preoperative serum CA125 level $\left(P=2.51 \mathrm{E}^{-4}\right)$ as independent risk predictors of ovarian involvement (Table 3). Other preoperative risk factors, including age at diagnosis, preoperative BMI, preoperative pathological tumor grade, tumor in 
specific-site, preoperative tumor size and peritoneal lavage cytology, did not appear associated with ovarian malignant involvement.

Finally, ROC curves were generated with preoperative myometrial invasion depth, the gross appearance of the ovaries, preoperative serum CA125 level, and a combination of these factors. The area under curve (AUC) were 0.692 (95\% confidence interval [CI], 0.563 to 0.821 ) for preoperative myometrial invasion depth alone group, 0.754 (95\% confidence interval [Cl], 0.621 to 0.887 ) for the gross appearance of the ovaries alone group, and 0.756 (95\% confidence interval [CI], 0.624 to 0.888 ) for preoperative serum CA125 level alone group, and 0.858 ( $95 \%$ confidence interval [Cl], 0.757 to 0.960 ) for the combination group, respectively. The combination of preoperative myometrial invasion depth, the gross appearance of the ovaries, and preoperative serum CA125 level (Figure 2) displayed a strong intensity predictive value for postoperative ovarian malignant involvement in premenopausal clinical stage I endometrioid endometrial carcinoma patients.

\section{Discussion}

A considerable proportion of premenopausal women are diagnosed with endometrioid endometrial carcinoma, the majority of who are classified as preoperative clinical stage I [4]. Younger premenopausal patients with clinical I stage endometrioid endometrial cancer have a more favorable prognosis than postmenopausal patients. An earlier large-scale study reported a 5-year disease-specific survival rate of $93 \%$ in women younger than 40 years [11]. The higher survival of younger patients may be partly attributed to early-stage and low-grade tumors.

For patients with clinical stage I endometrioid endometrial carcinoma, the traditional surgical approach is total hysterectomy and BSO with or without pelvic and/or para-aortic lymph node dissection according to the existence of high-risk factors. Young premenopausal women with clinical stage I endometrioid endometrial carcinoma subjected to total hysterectomy and BSO often not only suffer from permanent loss of fertility but also experience climacteric symptoms of decreased estrogen production. Hence, ovarian preservation is a feasible option for this group of patients in the absence of high risk factors. Wright and co-workers confirmed the safety and feasibility of ovarian preservation in a population-based analysis [12]. Consistently, Lee et al. [7, 13] reported that ovarian preservation in premenopausal women with early-stage endometrial carcinoma was not associated with poorer outcomes. Another study by Richter and colleagues showed that BSO not only induced better disease-free survival but also did not affect overall survival in young endometrial carcinoma patients [14]. Jia and Zhang recently performed a meta-analysis of comparing overall survive between BSO group (9376 cases) and ovary preservation group (1340 cases) in 10716 patients from four studies from the USA, Korea, and China. They demonstrated that ovary preservation can significantly improve the overall survive and did not adversely impact the reduced recurrence-free survival in pre-menopausal patients with early-stage endometrial cancer. Although the patients with ovary preservation had a 2.1 folds risk of recurrence, they obtained a higher overall survive probability in the long run [15]. 
However, occasionally these patients present with occult ovarian malignant involvement, as evident from postoperative detailed pathological inspection. In our analysis, ovarian malignant involvement occurred in $4.5 \%$ patients with clinical stage I endometrioid endometrial carcinoma, in keeping with Lin et al. [16] who reported ovarian involvement in $5 \%$ of their patient group. The ratio of ovarian malignant involvement in low risk patients was $1.04 \%$ while patients in the sub-high risk group positive for at least one risk factor showed higher ovarian involvement (6.6\%). Data from Kaplan-Meier analysis in the current study showed longer disease-free and higher overall survival rates in patients without ovarian malignant involvement relative to those with ovarian involvement. Ovarian malignant involvement, representing an advanced stage of disease, is generally associated with poorer prognosis. Effective ways to identify patients at higher risk of ovarian malignant involvement and preoperative risk factors that can be used to predict ovarian malignant involvement, especially to distinguish the subgroups of patients suitable for receiving staging surgery for endometrioid endometrial cancer, remains an urgent requirement. The majority of studies to date have focused on postoperative pathological prediction factors, such as myometrial invasion, tumor size, lymphovascular involvement, tumor grade, lymph node metastasis and cervical invasion. Sun and co-workers reported adnexal morphology, lymph node involvement (confirmed via frozen sections) and tumor spread in the peritoneal cavity as the most significant predictors of ovarian involvement, based on data from 203 young women with early-stage endometrial cancer [3]. Chen et al. [17] utilized postoperative histological and pathologic data (tumor size, histological type, pathological grade, peritoneal cytology, invasive depth of myometrium, uterine serosal involvement, lymph vascular space invasion, cervical involvement, and adnexa involvement) as effective parameters for distinguishing synchronous primary cancers of endometrium and ovary and endometrial cancer with metastasis to the adnexa. Clinicopathological characteristics (i.e., tumor size, myometrial invasion, lymphovascular space involvement, lymph node metastasis, tumor grade, cervical invasion and ovarian enlargement $\geq 5 \mathrm{~cm}$ ) were used to assess the likelihood of ovarian malignancy by Yoshino and colleagues, who showed the presence of ovarian metastasis in $4.5 \%$ patients and identified lymph node metastasis and deep myometrial invasion as significant predictive factors for ovarian metastasis and lymph node metastasis, respectively [18]. Li et al. [19] highlighted specific post-operation parameters, such as deep myometrial invasion, positive lymph node metastasis, positive LVSI, and high histologic grade (G2-G3), associated with ovarian involvement in younger endometrial cancer patients. Furthermore, in multivariate analysis, only deep myometrial invasion was an independent risk factor for ovarian involvement. However, the information is meaningless, because all the above risk factors were evaluated following the operation and not useful for gynecologists prior to surgery. Here, we focused on identifying potential preoperative predictive factors of ovarian malignant involvement, with the aim of providing beneficial guidelines for the appropriate surgical interventions.

Our results suggested that preoperative imaging of myometrial invasion depth, the gross appearance of the ovaries, and preoperative serum CA125 level, were predictive risk factors of ovarian malignant involvement in premenopausal clinical stage I endometrioid endometrial carcinoma patients, which could aid in preoperative counseling of patients and clinical decision-making for the first time. Univariate and multivariate logistic analyses further validated preoperative imaging of myometrial invasion depth, the 
gross appearance of the ovaries, and preoperative serum CA125 as independent risk predictors of ovarian involvement in our patient group. Consistently, AUC data showed that combination of preoperative myometrial invasion depth, the gross appearance of the ovaries, and preoperative serum CA125 level had strong predictive value for postoperative ovarian malignant involvement to a degree. Preoperative deep myometrial invasion depth and serum CA125 level have been previously identified as prognostic factors in ovarian metastasis [20]. Yu et al. found the preoperative imaging of myometrial invasion depth by magnetic resonance imaging had the values for diagnostic sensitivity $(69.2 \%)$, specificity (80\%), positive predictive value (50\%), and negative predictive value $(90 \%)$, with postoperative pathologic assessment used as the reference standard in a total of 58 endometrial carcinoma cases research [21]. Our study results are in accordance with them, with lower sensitivity (52.94\%) and higher specificity (97.27\%). Even though, the preoperative imaging of myometrial invasion depth still has medium preoperative predictive value for postoperative ovarian malignant involvement due to its higher specificity. Furhtermore, Jiang and colleagues reported that preoperative serum CA125 is an effective predictor of lymph node metastasis in patients with endometrial cancer, in particular, clinical stage I [22]. Analysis of the combined factors also revealed utility as a predictive marker of ovarian malignant involvement to some extent. CA125 has been applied as a tumor marker of ovarian carcinoma since its discovery 30 years ago [23]. A large proportion (80\%) of women with primary epithelial ovarian carcinoma and secondary ovarian tumor $(70 \%)$ are diagnosed based on elevated CA125 levels [24, 25]. Taken together, our data suggest that the combination of preoperative myometrial invasion depth, the gross appearance of the ovaries and preoperative serum CA125 level may present an effective predictive risk factor of postoperative ovarian malignant involvement in patients with clinical stage I endometrioid endometrial carcinoma.

Our study had several of limitations that should be acknowledged. One significant factor was the origin of ovarian tumor. Although we set stricter clinicopathological criteria for diagnosis, classification of a part of patients into ovarian malignant involvement or simultaneous uterine and ovarian carcinoma groups was difficult. Moreover, precursor lesions, such as endometrial hyperplasia or concurrent ovarian endometriosis, were not consistently addressed in pathology reports, potentially affecting the final diagnosis. Therefore, novel powerful genetic tools require development for accurate classification of patients displaying complex symptoms in forthcoming research, which would lead to a more accurate research database. Second, large-scale prospective clinical studies are necessary to ascertain whether the benefits of ovarian preservation outweigh the risks of surgical procedures in clinical stage I endometrioid endometrial carcinoma to reduce bias.

\section{Conclusions}

The main purpose of the present study was to identify preoperative predictive factors of ovarian malignant involvement in premenopausal patients with clinical stage I endometrioid endometrial carcinoma. Our results showed that the incidence of ovarian malignant involvement in these patients is relatively minimal. A combination of preoperative myometrial invasion depth, the gross appearance of the ovaries, and preoperative serum CA125 level appeared to have strong predictive value for postoperative ovarian malignant involvement and may thus aid in informed decision-making on whether or not ovarian 
preservation should be performed in premenopausal patients with clinical stage I endometrioid endometrial carcinoma.

\section{Abbreviations}

FIGO: International Federation of Gynecology and Obstetrics; LVSI: lymph vascular space invasion; LNM: lymph node metastasis; BMI: Body Mass Index; CA125: Carbohydrate Antigen 125; DFS: disease-free survival; OS: overall survival; BSO: bilateral salpingo-oophorectomy; ROC curve: receiver operating characteristic curve; AUC: area under curve; TPR: true positive rate; TNR: true negative rate; PPV: positive predictive value; NPV: negative predictive value.

\section{Declarations}

\section{Ethics approval and consent to participate}

The present study was approved by the Institutional Review Board of Women's Hospital, Zhejiang University School of Medicine. Written, informed consent has been given and obtained from all individual participants included in the study.

\section{Consent to publish}

Not applicable.

\section{Availability of data and materials}

All data generated and /or analyzed during the current study are available from corresponding author on reasonable request.

\section{Competing interests}

The authors declare no conflict of interest.

\section{Funding}

This study was supported by Zhejiang Provincial Natural Science Foundation of China (Grant No. LY18H160004).

\section{Authors' contributions}

Conceived and designed the study: BHL. Data collection and analysis: QC and BHL. Pathological sections confirmed: QC and WWW. Preparation of the manuscript: YF and QC. Reviewed and commented on the manuscript: WGL and BHL. All authors read and approved the final manuscript.

\section{Acknowledegements}




\section{References}

1. Burke WM, Orr J, Leitao M, Salom E, Gehrig P, Olawaiye AB, Brewer M, Boruta D, Villella J, Herzog T et al: Endometrial cancer: a review and current management strategies: part I. Gynecologic oncology 2014, 134(2):385-392.

2. Burke WM, Orr J, Leitao M, Salom E, Gehrig P, Olawaiye AB, Brewer M, Boruta D, Herzog TJ, Shahin FA: Endometrial cancer: a review and current management strategies: part II. Gynecologic oncology 2014, 134(2):393-402.

3. Sun C, Chen G, Yang Z, Jiang J, Yang X, Li N, Zhou B, Zhu T, Wei J, Weng D et al: Safety of ovarian preservation in young patients with early-stage endometrial cancer: a retrospective study and metaanalysis. Fertility and sterility 2013, 100(3):782-787.

4. Soliman PT, Oh JC, Schmeler KM, Sun CC, Slomovitz BM, Gershenson DM, Burke TW, Lu KH: Risk factors for young premenopausal women with endometrial cancer. Obstetrics and gynecology 2005, 105(3):575-580.

5. Lewin SN: Revised FIGO staging system for endometrial cancer. Clinical obstetrics and gynecology 2011, 54(2):215-218.

6. Creasman WT, Morrow CP, Bundy BN, Homesley HD, Graham JE, Heller PB: Surgical pathologic spread patterns of endometrial cancer. A Gynecologic Oncology Group Study. Cancer 1987, 60(8 Suppl):2035-2041.

7. Lee TS, Jung JY, Kim JW, Park NH, Song YS, Kang SB, Lee HP: Feasibility of ovarian preservation in patients with early stage endometrial carcinoma. Gynecologic oncology 2007, 104(1):52-57.

8. Ulbright TM, Roth LM: Metastatic and independent cancers of the endometrium and ovary: a clinicopathologic study of 34 cases. Human pathology 1985, 16(1):28-34.

9. Turashvili G, Gomez-Hidalgo NR, Flynn J, Gonen M, Leitao MM, Jr., Soslow RA, Murali R: Risk-based stratification of carcinomas concurrently involving the endometrium and ovary. Gynecologic oncology 2019, 152(1):38-45.

10. Mariani A, Webb MJ, Keeney GL, Haddock MG, Calori G, Podratz KC: Low-risk corpus cancer: is lymphadenectomy or radiotherapy necessary? American journal of obstetrics and gynecology 2000, 182(6):1506-1519.

11. Matsuo K, Machida H, Frimer M, Marcus JZ, Pejovic T, Roman LD, Wright JD: Prognosis of women with stage I endometrioid endometrial cancer and synchronous stage I endometrioid ovarian cancer. Gynecologic oncology 2017, 147(3):558-564.

12. Wright JD, Buck AM, Shah M, Burke WM, Schiff PB, Herzog TJ: Safety of ovarian preservation in premenopausal women with endometrial cancer. Journal of clinical oncology : official journal of the American Society of Clinical Oncology 2009, 27(8):1214-1219. 
13. Lee TS, Lee JY, Kim JW, Oh S, Seong SJ, Lee JM, Kim TJ, Cho CH, Kim SM, Park CY: Outcomes of ovarian preservation in a cohort of premenopausal women with early-stage endometrial cancer: a Korean Gynecologic Oncology Group study. Gynecologic oncology 2013, 131(2):289-293.

14. Richter CE, Qian B, Martel M, Yu H, Azodi M, Rutherford TJ, Schwartz PE: Ovarian preservation and staging in reproductive-age endometrial cancer patients. Gynecologic oncology 2009, 114(1):99-104.

15. Jia $P$, Zhang $Y$ : Ovarian preservation improves overall survival in young patients with early-stage endometrial cancer. Oncotarget 2017, 8(35):59940-59949.

16. Lin KY, Miller DS, Bailey AA, Andrews SJ, Kehoe SM, Richardson DL, Lea JS: Ovarian involvement in endometrioid adenocarcinoma of uterus. Gynecologic oncology 2015, 138(3):532-535.

17. Chen L, Zhao Q, Lv X: Characteristics and prognosis of coexisting adnexa malignancy with endometrial cancer: a single institution review of 51 cases. Archives of gynecology and obstetrics 2011, 283(5):1133-1137.

18. Kinjyo Y, Kudaka W, Ooyama T, Inamine M, Nagai Y, Aoki Y: Ovarian preservation in young women with endometrial cancer of endometrioid histology. Acta obstetricia et gynecologica Scandinavica 2015, 94(4):430-434.

19. Li J, Zhu Q, Yang B, Ning C, Liu X, Luo X, Chen X: Risk factors for ovarian involvement in young and premenopausal endometrioid endometrial cancer patients. European journal of obstetrics, gynecology, and reproductive biology 2018, 222:151-154.

20. Kubecek O, Laco J, Spacek J, Petera J, Kopecky J, Kubeckova A, Filip S: The pathogenesis, diagnosis, and management of metastatic tumors to the ovary: a comprehensive review. Clinical \& experimental metastasis 2017, 34(5):295-307.

21. Guo Y, Wang P, Wang P, Gao W, Li F, Yang X, Ni H, Shen W, Guo Z: Myometrial invasion and overall staging of endometrial carcinoma: assessment using fusion of T2-weighted magnetic resonance imaging and diffusion-weighted magnetic resonance imaging. OncoTargets and therapy 2017, 10:5937-5943.

22. Jiang T, Huang L, Zhang S: Preoperative serum CA125: a useful marker for surgical management of endometrial cancer. BMC cancer 2015, 15:396.

23. Bottoni P, Scatena R: The Role of CA 125 as Tumor Marker: Biochemical and Clinical Aspects. Advances in experimental medicine and biology 2015, 867:229-244.

24. de Waal YR, Thomas CM, Oei AL, Sweep FC, Massuger LF: Secondary ovarian malignancies: frequency, origin, and characteristics. International journal of gynecological cancer : official journal of the International Gynecological Cancer Society 2009, 19(7):1160-1165.

25. Yang WL, Lu Z, Bast RC, Jr.: The role of biomarkers in the management of epithelial ovarian cancer. Expert review of molecular diagnostics 2017, 17(6):577-591.

\section{Tables}

Table 1 Postoperative demographics and clinicopathological data of 511 premenopausal patients. 


\begin{tabular}{|c|c|c|}
\hline Characteristics & No. of patients & Percentage (\%) \\
\hline \multicolumn{3}{|l|}{ Patient age (year) } \\
\hline$\leq 40$ & 121 & $23.68 \%$ \\
\hline$\square 40$ and $\leq 50$ & 390 & $76.32 \%$ \\
\hline \multicolumn{3}{|l|}{ FIGO stage } \\
\hline$<$ II & 384 & $75.15 \%$ \\
\hline$\geq \mathrm{II}$ & 127 & $24.85 \%$ \\
\hline \multicolumn{3}{|l|}{ Differentiation } \\
\hline Well & 363 & $71.04 \%$ \\
\hline Moderate & 107 & $20.94 \%$ \\
\hline Poor & 41 & $8.02 \%$ \\
\hline \multicolumn{3}{|l|}{ Myometrial invasion } \\
\hline$\square 1 / 2$ & 477 & $93.34 \%$ \\
\hline$\geq 1 / 2$ & 34 & $6.65 \%$ \\
\hline \multicolumn{3}{|l|}{ Lymphadenectomy } \\
\hline Yes & 318 & $62.23 \%$ \\
\hline No & 193 & $37.77 \%$ \\
\hline \multicolumn{3}{|l|}{ LNM } \\
\hline Yes & 16 & $5.03 \%$ \\
\hline No & 302 & $94.97 \%$ \\
\hline \multicolumn{3}{|c|}{ Cervical stromal involvement } \\
\hline Yes & 92 & $18.01 \%$ \\
\hline No & 419 & $81.99 \%$ \\
\hline \multicolumn{3}{|l|}{ LVSI } \\
\hline Yes & 15 & $2.94 \%$ \\
\hline No & 496 & $97.06 \%$ \\
\hline \multicolumn{3}{|l|}{ Ovarian involvement } \\
\hline Yes & 23 & $4.50 \%$ \\
\hline No & 488 & $95.50 \%$ \\
\hline
\end{tabular}

FIGO, International Federation of Gynecology and Obstetrics; LVSI, lymph vascular space invasion; LNM, lymph node metastasis. 
Table 2 Univariate logistic analysis showed preoperative predictive factors of ovarian malignant involvement in 511 premenopausal patients.

\begin{tabular}{|c|c|c|c|}
\hline \multirow[t]{2}{*}{ Characteristics } & \multicolumn{2}{|c|}{ Ovarian malignant involvement, n (\%) } & \multirow[t]{2}{*}{$P$-value } \\
\hline & No & Yes & \\
\hline \multirow[t]{2}{*}{ Patient age (year) } & & & 0.671 \\
\hline & $43.93 \pm 5.52$ & $43.26 \pm 7.41$ & \\
\hline Preoperative BMI $\left(\mathrm{Kg} / \mathrm{m}^{2}\right)$ & & & 0.381 \\
\hline$<30$ & $443(86.7)$ & $22(4.3)$ & \\
\hline$\geq 30$ & $45(8.8)$ & $1(0.2)$ & \\
\hline Preoperative pathological tumor grade & & & 0.158 \\
\hline Well/moderate & $463(90.6)$ & $20(3.9)$ & \\
\hline Poor & $25(4.9)$ & $3(0.6)$ & \\
\hline Tumor in specific-site & & & 0.422 \\
\hline No & $449(87.9)$ & $20(3.9)$ & \\
\hline Yes & $39(7.6)$ & $3(0.6)$ & \\
\hline Preoperative myometrial invasion depth & & & $1.0 \mathrm{E}^{-6}$ \\
\hline$<1 / 2$ & $466(91.2)$ & $14(2.7)$ & \\
\hline$\geq 1 / 2$ & $22(4.3)$ & $9(1.8)$ & \\
\hline Preoperative tumor size (cm) & & & 0.111 \\
\hline$<4$ & $407(79.6)$ & $16(3.1)$ & \\
\hline$\geq 4$ & $81(15.9)$ & $7(1.4)$ & \\
\hline Peritoneal lavage cytology & & & 0.107 \\
\hline Negative & $486(95.1)$ & $22(4.3)$ & \\
\hline Positive & $2(0.4)$ & $1(0.2)$ & \\
\hline The gross appearance of the ovaries & & & $1.0 \mathrm{E}^{-5}$ \\
\hline Normal & $426(83.4)$ & $10(2.0)$ & \\
\hline Abnormal & $62(12.1)$ & $13(2.5)$ & \\
\hline \multirow[t]{2}{*}{ Preoperative serum CA125 (U/L) } & & & $3.2 \mathrm{E}^{-5}$ \\
\hline & $22.65(0.4-392)$ & $135.2(1.0-834)$ & \\
\hline
\end{tabular}

BMI, Body Mass Index; CA125, Carbohydrate Antigen 125. 
Table 3 Multivariate logistic analysis showed preoperative predictive factors of ovarian malignant involvement in 511 premenopausal patients.

\begin{tabular}{lcccc}
\hline \multicolumn{1}{c}{ Characteristics } & Regression coefficient & Standard error & $\chi^{2}$ & $P$-value \\
& (B) & $(\mathrm{SE})$ & (Wald) & \\
\hline operative myometrial invasion depth & 1.099 & 0.395 & 7.763 & 0.005 \\
operative serum CA125 (U/L) & 0.014 & 0.004 & 13.405 & $2.51 \mathrm{E}^{-4}$ \\
gross appearance of the ovaries & 1.334 & 0.234 & 32.375 & $1.27 \mathrm{E}^{-8}$ \\
\hline
\end{tabular}

CA125, Carbohydrate Antigen 125.

\section{Figures}
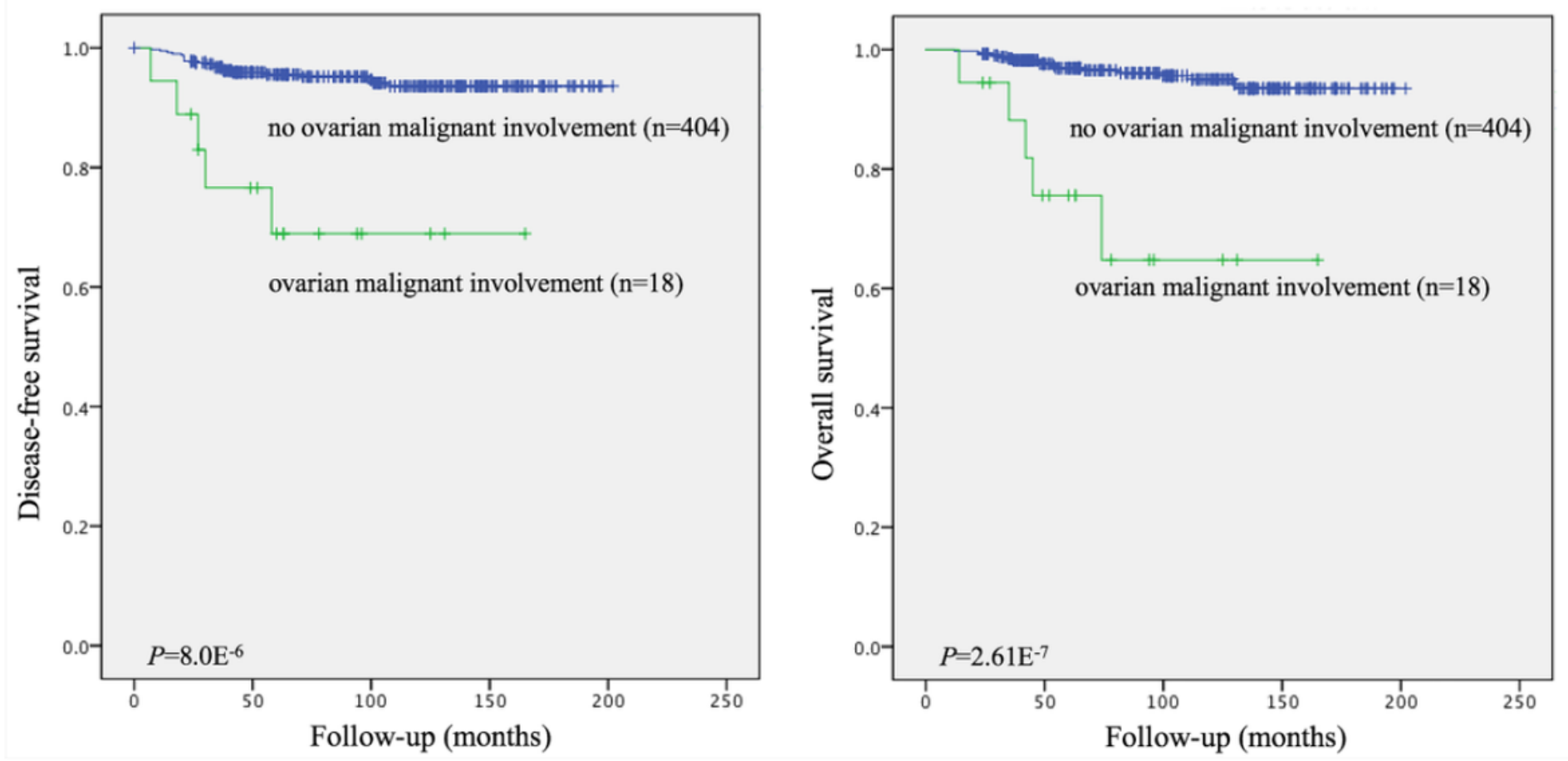

Figure 1

Kaplan-Meier curves showing the relationship between status of ovarian malignant involvement and disease-free survival (DFS) and overall survival (OS) in 422 premenopausal patients with clinical stage I endometrioid endometrial carcinoma. The patients with ovarian malignant involvement were significantly associated with shorter DFS (A) and OS (B). 


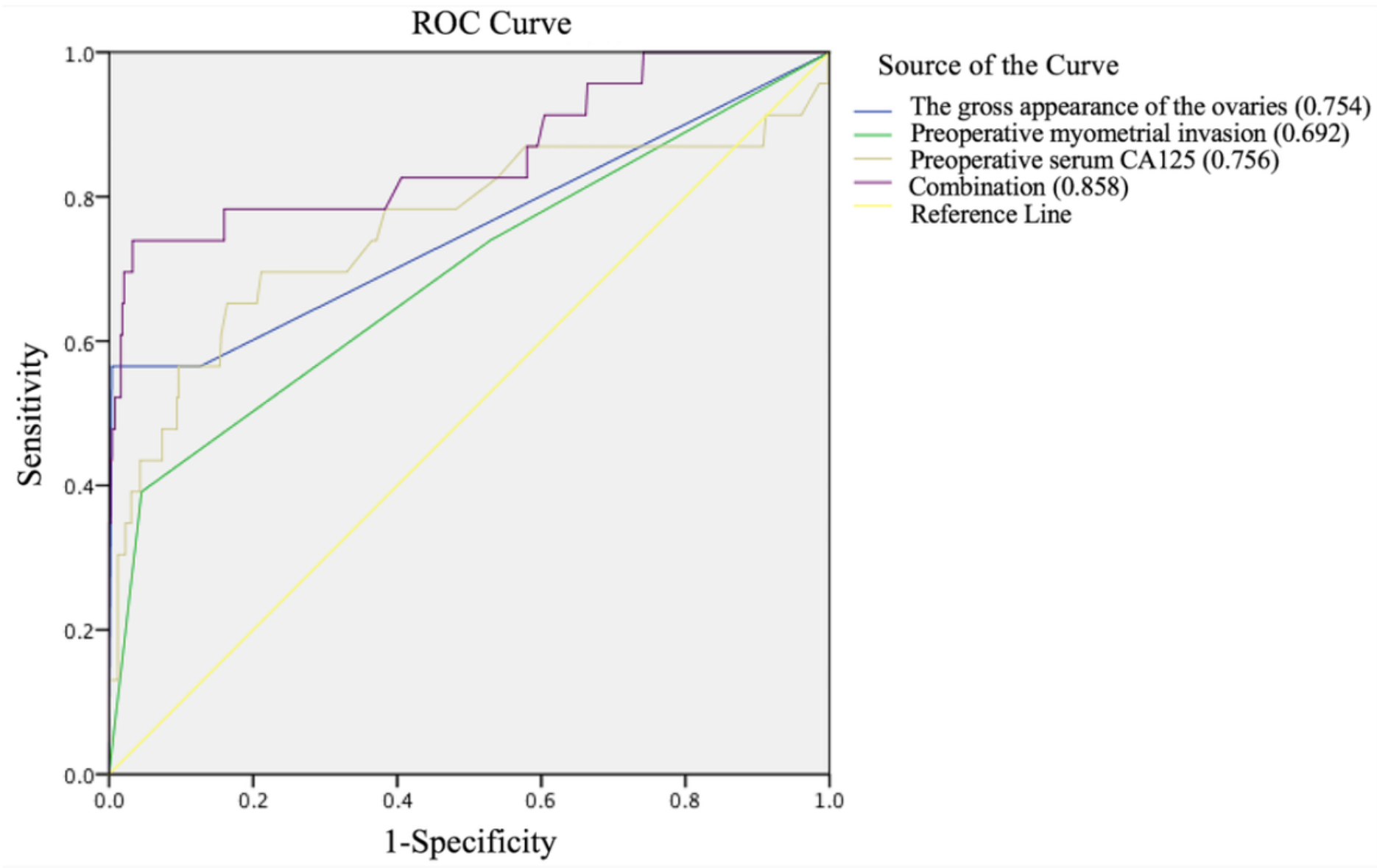

Figure 2

AUC of the multivariate base model (the combination of preoperative serum CA125 level, the gross appearance of the ovaries, and preoperative myometrial invasion depth) could have strong predictive value for postoperative ovarian malignant involvement in 511 premenopausal patients with clinical stage I endometrioid endometrial carcinoma. 